\title{
Perception of Feeding Practices in Home Management of Childhood Diarrhoea in Ibadan North East Local Government Area, Oyo State, Nigeria
}

\section{Lukman Tunde Fasasi Ayodele Samuel Jegede}

Department of Sociology University of Ibadan Ibadan, Nigeria

DOI:10.36108/NJSA/7102/51(0230)

\begin{abstract}
Diarrhoea accounts for one in five deaths amongst children in Nigeria. In home management of childhood diarrhoea, cultural diversity exists in people's perceptions of appropriate feeding practices during and after diarrhoeal episodes. This study examined factors that influenced people's perceptions of feeding practices in home management of childhood diarrhoea in Ibadan North East Local Government Area of Oyo state, using Weberian social action theory. The study design was exploratory using qualitative techniques. Sixteen (16) In-depth interviews (IDIs) for mothers of under-five children, six (6) Key Informant Interviews (KIIs) for Community leaders, Herbalist and Nurses in public health institutions, and four (4) Focus Group Discussions were conducted. Teething, poor sanitation and consumption of some foods were perceived as responsible for high prevalence of diarrhoea amongst children. Mothers' perceptions of type of food to be served to children, and number of feeding times, during and after diarrhoeal episodes varied according to their social status and food culture of their local environment. The study concluded that mothers' perceptions of feeding practices in home management of childhood diarrhoea were influenced by culture, religion and socio-demographic factors. Injurious beliefs should therefore be dropped towards enhancing success of primary health care (PHC) programme.
\end{abstract}

Keywords: Diarrhoea, feeding practices, perception, home management

\section{Introduction}

Diarrhoea is a health condition in which a child has 3 or more loose or liquid stools per day, or described as having more stools than is normal for that person (World Health Organisation, W.H.O, 2011). Worldwide, diarrhoea claims the life of 2 million children each year, $22 \%$ of which occur in SubSaharan Africa (Mwambette and Joseph, 2010). It is the second most important cause of childhood mortality in sub-Sahara Africa (SSA), and responsible for the death of one in 12 children (United Nations' Children, Education, and Scientific Fund, UNICEF/WHO, 2009). It is estimated to have killed more children around the World than malaria, Acquired Immunodeficiency Syndrome (AIDS) and tuberculosis combined (Mwanbete and Joseph, 2010).

Report by UNICEF (2009) indicates that more than one in every 5 children in Nigeria dies before age five, and that as high as $40 \%$ of these deaths are due 
to diarrhoea. Most deaths from diarrhoea are often due to loss of body fluid through dehydration. Diarrhoea is a major cause of morbidity and mortality in rural communities in Nigeria (Collise and Goduka, 2012). Mortality from diarrhoeal condition disproportionately targets individuals in low and middleincome countries, where food insecurity is highly prevalent (Food Agriculture Organisation, FAO, 2011). This could largely be due to the living condition in most communities in Sub-Saharan Africa. Poverty has been considered as the major predisposing factor to childhood diarrhoea in Sub Saharan Africa (United Nations Development Programme, UNDP, 2010). Living in socioeconomic-deprived neighbourhoods is reported to be associated with less likelihood of seeking medical care (Aremu, Lawoko, Moradi and Dalal, 2011). The well-being of a child is determined by multiple environmental factors which include food insecurity (lack of money to purchase enough nutritious food), energy availability (insufficient energy for proper growth) (Children's health watch, 2012).

Shuaib, Nigel and Jan Van den Broeck (2004) identified eleven 'local types of diarrhoea', which were classified into three categories based on their perceived causes: (a) natural causation (b) super natural causation, and (c) those caused by 'germs' or change in diet. In some cultures, especially amongst the Yoruba of South-western Nigeria, diarrhoea is believed to be caused by two factors: natural and man induced (Asakitipi, 2007). The type of diarrhoea linked with childhood teeth formation is known as 'Igbe-gbuuru' and considered to be natural, while man-induced diarrhoea is called 'igbe jedi-jedi which is linked with dietary habit, such as consumption of sugary foods. While some preventive measures are taken against diarrhoeas that are believed to be man-induced, the same is not the case for diarrhoeas that are naturally induced. This perception explains the high morbidity and mortality rates amongst the under-fives (Asakitipi, 2007).

Mothers' knowledge and perceptions of childhood diarrhoea, as caregivers, often influenced their choice of treatment, and thus related to mortality and morbidity (Ghasemi, Talebian, Masaoud and Mousari, 2013). In a study by Ogunbiyi and Akinyele (2010), mothers adopt combination of treatments in managing episode of diarrhoea. There is a high perception of the diverse benefits and effectiveness of complementary alternative medicine, such as herbs (Okoronkwo et al., 2014). This has led to reliance on indigenous medicinal plants as cheaper alternative in the treatment and management of childhood diarrhoea (Collise and Goduka, 2012).

According to a report published by Adhikari (2010) on Food utilization, beliefs and taboos, feeding during illnesses receive insufficient or no attention during common childhood illnesses such as diarrhoea and acute respiratory infections. Classification of foods was based on food beliefs, as pure and impure food; hot, cold and neutral foods; beneficial or harmful foods; or curative foods (Adhikari, 2010). This often leads to food restrictions and withdrawals. The type of food restriction/withdrawal depends to a large extent on the perception of the mothers, and the food culture of the local community. 
Most food substances commonly withdrawn are solid foods such as fruits, meat products, milk products and vegetables (Ansari, Ibrahim, Hassali, Shankar, Koirala, Thapa, 2012). Childhood diarrhoea is an important cause of malnutrition which can be worsened when caregivers limit nutritional support to the affected child (Pantenburg, 2014).

There are cultural factors which often influence choice of food and feeding patterns of children during diarrhoea episodes. Food taboos are based on people's culture (Jegede, Oke, Oluwatosin, Ajala, and Adejumo, 2002). There are taboos against certain food which affects maternal nutrition during lactation (Adhikari, 2010). Food intakes of nursing mothers impact on mother to child health transmission. Mothers decide on type of food to be given to children, during and after sickness, and the overall management of the disease (Merga and Alemayehu, 2015).

Ethnicity also influenced mother's perceptions as it determines people's perception of illness and diseases. According to Fayehun and Omololu (2008), this substantiates the fact that mother's ethnicity and its inherent socio-cultural practices significantly predispose her child to higher risk of childhood diarrhoea if other factors are held constant. Yilgwan, and Okolo (2012), in their study on prevalence of diarrhoea disease and risk factors in Jos University Teaching Hospital, Nigeria, demonstrated an important relationship between diarrheal morbidity and low maternal education, nonexclusively breastfed infant, and previous diarrheal episode in a sibling. Thus ethnicity, source of drinking water, age of child and educational level of mothers are important determinants of childhood diarrhoea.

However, Ogunbiyi and Akinyele (2010) further reveals a low knowledge of nutritional management of diarrhoea diseases by families. Thus, according to UNICEF and WHO, Family knowledge about diarrhoea must be reinforced in such areas as prevention, nutrition, ORT/ORS use, zinc supplementation, and when and where to seek care (WHO/UNICEF, 2003).

Many studies have been conducted on diarrhoea in Nigeria. Fayehun and Omololu (2008) examined the prevalence of childhood diarrhoea and various treatment methods adopted by different ethnic groups in Nigeria, Asakitipi (2007) examined the concept of diarrhoea among Yoruba women in Ibadan, and Asakitipi (2010) further examined the influence of mother's knowledge about ORT on its usage. However, there appeared to be limited research on knowledge and perception of feeding practices during and after episodes of childhood diarrhoea. This study examined people's knowledge and perception about feeding practices of under-five children in the management of childhood diarrhoea.

This study adopted Weberian social action theory to discuss mothers' subjective meanings about causes and cure of diarrhoea, the roles of culture and significant others on perceptions about home management of childhood diarrhoea. 


\section{Materials and Methods}

\section{Research design and Study Area}

The research design for this study was exploratory, using qualitative data. The study was carried out in eight (8) communities namely: Basorun, Iwo road, Labiran, Aromolaran, Ojaagbo, Aremo, Oranyan and Orita-aperin, in Ibadan North East Local Government Area of Oyo State. The communities were randomly selected from the twelve (12) politically delineated wards in the LGA. The inhabitants are predominantly Yoruba with significant presence of people of other tribes and nationalities. It is highly heterogeneous. The area was chosen because of the presence of Public health institutions in all the wards. There is poor supply of pipe borne water from the state, and supplemented with water from wells and streams like Ogunpa.

The study population was limited to Yoruba women of reproductive age with, or who had experienced the care of, under-five children as at the time of the study, and who were permanent residents of the study area. Mothers were chosen because of their roles in provision of health care and food for children. Participants for In-depth interviews (IDIs) were selected in houses with mothers with under-five children, and the readily available mothers were chosen, and where there was none the next household was considered as a replacement. Participants for key informant interviews (KIIs) were selected from public hospitals, community leaders and herbal homes in the study areas. Matrons of the public hospitals were selected, while community leaders were chosen, based on referrals, from those recognised as leaders of influence by their people,

Participants for the FGDs were selected on the basis of literacy and gender. Those who were not educated were grouped together while those who were educated were similarly grouped, and separated into two groups each of male and female, literate and illiterate. Data collection involved triangulation of three qualitative methods of Focus Group Discussions (FGDs), In-depth interviews (IDIs) and Key Informant Interviews (KIIs). Sixteen (16) IDIs, 2 from each of the 8 selected wards in the LGAs, were undertaken with mothers of under-five children; Six KIIs were carried out, 2 each for Community leaders, Herbalist and Nurses (Matrons) in public health institutions. Four (4) FGDs were conducted, each made up of 8-10 participants, amongst literate nursing mothers with under-five children, illiterate nursing mothers with under-five children, literate fathers with under-five children and illiterate fathers with under-five children. All interviews for illiterate participants were conducted in Yoruba language, while those for literate participants were conducted in English language.

The interviews and FGDs focused on eliciting information about people's knowledge and belief, about causes of childhood diarrhoea, and their perceptions of feeding practices, during and after episodes of childhood diarrhoea. It also involved discussions on factors that influenced choice of treatment option, in the selected households, on management of childhood diarrhoea. The recorded interviews and discussions were transcribed. The 
recordings done in Yoruba were translated into English language, and transcribed for subsequent analysis. The transcriptions were compared with the notes taken during data collection. The data collected were content and thematically analysed, to identify and group similar thoughts or distinct opinions expressed across the three methods. This was done according to the objectives of the study. Information obtained was reported as phrases in quotes from the recorded expressions of participants. Internal validity was achieved by using data from all the three sources, and by presenting views from different participants to support the research objectives.

The instruments used for data collection were interview and FGD guides designed for each group of participants. These however did not exclude interviewers' discretion within the scope of the study to facilitate obtaining detailed and relevant information on the objectives of the study. Participants were selected based on principle of confidentiality of data, beneficence to participant, Non-Malfeasance to participants and Voluntariness.

\section{Results}

\section{Knowledge, Perception and Belief about Childhood Diarrhoea}

The study attempted to obtain data on people's knowledge, perceptions and beliefs about childhood diarrhoea. On knowledge of the causes of childhood diarrhoea, most of the respondents expressed that diarrhoea could be caused by teething in under-five children. This was the dominant position of most participants during FGDs. An excerpt from the view of a participant on the people's perceptions of the causes of diarrhoea is transcribed below:

It is normal when a child is growing teeth. Teething is a major cause of diarrhoea since it is strange to the body of the child. All my children experienced it in the past and this is often characterised by slight increase in body temperature and subsequent loss of appetite. This goes away as soon as the tooth shows up. It is often more intense and take longer days if the number of teeth is more than one or it is the premolars. (FGD/Literate mothers/Basorun)

Another participant while explaining how advent of tooth could cause diarrhoea described the behaviour of a child during teething that could cause diarrhoea:

It (diarrhoea) is often caused by advent of tooth which can be noticed with the child putting dirty things in the mouth. The gum is always itching and the child will attempt to scratch it with dirty things like mud, sand or even sticks which could result to stooling due to infection. (FGD/Illiterate mothers/Aremo) 
However, some of those interviewed attributed the cause of childhood diarrhoea to poor food culture like poor feeding habit, with reference to poor food handling and food taboos in terms of proscriptions and prescriptions. One of such views was expressed by a participant during an IDI who stated thus:

Teething and eating of certain foods like ewa (beans), asaro (porridge), vegetables and hot food in the night are part of the causes of frequent stooling. (IDI/Nursing mother/27 years/ Aremo)

Some attributed the cause of childhood diarrhoea to the food intake of mothers. The opinion of an herbal practitioner during KII supported this position:

When mothers eat too much vegetable, they pass this to their children that are being breastfed, and this may not be tolerated by the children and thus lead to frequent stooling. Another cause may be when a pregnant woman is still breast feeding a child. (KII/Herbalist/Labiran)

A male participant during a FGD session stated that:

It is the mothers whose cook determines whether a child will stool or not. We pay them to cook good food but they decide what to cook. (FGD/Illiterate fathers/Orita-aperin)

On perception about fatality of diarrhoea, level of education was found to influence the opinions of participants. An educated participant during a FGD session conducted for literate fathers stated that:

Diarrhoea can kill, if a child continues to stool uncontrollably. This will lead to loss of body fluid, and the child will become lean and unable to fight any other disease. The immunity will break down. (FGD/Literate Fathers/Basorun)

However, the opinions of some participants at the FGD session conducted amongst illiterate mothers showed their belief that diarrhoea could not kill. One of such opinions is presented thus:

Hmm!!! (Coughs) It is normal for my children to stool. I always get Baba Kobomoje (a local and traditional paediatrician) to do incision on the chin of my babies for them, and so they cannot die. (FGD/Illiterate mothers/Aremo)

Another participant at the same session gave a reason why she believed diarrhoea could not kill, and why the frequent passing out of stool should not be stopped:

No! No!! Diarrhoea cannot kill. I see it as a means by which children get rid of germs, bad or excess food they had eaten, from their systems. It is not good to stop it. (FGD/Illiterate mothers/Aremo) 


\section{Perceptions about ORS}

On mothers' awareness about the use of Oral Rehydration Therapy or Solution (ORT or ORS) in treatment of diarrhoea, opinions expressed during FGD session revealed that most of them often combined ORT with some other medications because of their perceptions about the efficacy of such practice in the management of childhood diarrhoea. The opinion of a nursing mother during an IDI summed up this perception:

Yes! I am aware of ORS, but I feel sometimes a simple mixture of salt and sugar will not be enough to take care of the stooling. So, I often preferred to go straight to the hospital. (IDI/Nursing mother/30 years/Basorun)

Some participants who engaged in self-medication expressed their beliefs in the efficacy of other medications other than ORT. One of such opinions was presented by a nursing mother:

I believe that stooling frequently requires more serious attention than just taking a mixture of salt and sugar. I often use flagyl tablet and tetracycline capsules as advised by my neighbours to quickly stop the stooling. (IDI/Nursing mother/27 years/ Ojaagbo)

An herbal medicine practitioner also supported the above claim during a KII, by also revealing the people's belief about the supernatural causes of childhood diarrhoea:

You need more than a simple salt solution to combat the germs and accumulation of dirt in the stomach of the child. We often use agbo (herbal mixtures) and sometimes gbere (incisions) to ward off the evils of measles that may be preceded by frequent stooling as symptom. (KII/Herbalist/Labiran)

\section{On Treatment Options}

People adopted various methods in the treatment of childhood diarrhoea. There were different factors that influenced the choice of treatment patterns during episodes of childhood diarrhoea. The treatment choice and forms included: Home remedies with herbal mixtures and/ or orthodox medicine, patronage of open medicine vendors, patronage of private or public health institutions, consulting faith healers, or a combination of two or more therapeutic options. The reasons for some of these therapeutic choices were presented by some participants during FGDs.

One of those who patronised public hospitals gave some reasons for the choice of a public health institution as follow:

In government hospitals the drugs are cheap and they have the necessary equipments. Though the nurses are unfriendly and 
some of their workers prefer to refer you to their own clinics... (FGD/Illiterate mothers/Basorun)

A participant during an interview provided another justification for the patronage of public hospitals:

Hmmm!!! I patronise a public hospital because it is located near our house, and cheap. Again if there are complications they will quickly refer you to Orita mefa (University College Hospital, Ibadan), where you will get better medical attention. (IDI/Nursing mother/24 years/Aromoloran)

This position was contrasted by a nursing mother during a different IDI:

Yah! In private hospitals, the cost may be high but you get value for your money. The Nurses are courteous and Doctors will listen to your complaint with rapt attention. (IDI/Nursing mother/30 years/Iwo road)

However, some mothers took to seek spiritual remedy based on their belief about the supernatural cause of childhood diarrhoea, and perceived efficacy of the treatment option. A nursing mother opened up that:

I visit the Iya Abiye (faith clinic) in our area because of my experience on the need for prayers as spiritual guide, given the history of birth deliveries in our family. We are all from the mission house. We were all born there. (IDI/Nursing mother/32 years/ Orita-aperin)

Some people however combined two or more treatment options. One of those who combined these therapeutic options gave reasons for it:

For me, oh! I always explore all options. I often combine herbal mixtures with modern medicine. The modern medicine is a quick action but does not always go deep into the body to completely heal or clear it of the sickness. Agbo (Herbal mixture) will always be more effective, on the long run, while modern medicines will only give immediate relief to the sick child' (IDI/Nursing mother/28 years/Ojaagbo).

A community leader, while corroborating this position, provided additional reason for combining the treatment options by the people:

Our people often combine more than one method of treatment. This is because of our belief that more than one method will be more effective in controlling diarrhoea. And the hospital people (health workers) sometimes are not friendly, despite the fact that it is often costly. (KII/Community leader/Ojaagbo) 
On decisions about place of treatment, most male respondents stated that women often took decisions on where to take care of their children. It was believed that the care of children, including their health, was the traditional role of women. This was corroborated by a male participant at FGD session:

Our women are the ones taking care of us all. They are our mothers, and naturally care for us. We men just pay the bills. What do I know about how the children spend their days or feel? (FGD/Illiterate fathers/ Orita-aperin)

However, a nursing mother in Oranyan expressed a contrary position as regards decision making on place of treatment:

The decision as regards where to treat my child is always a family decision. Most often we rely on the advice of my motherin-law as an experienced person in the care of children. (IDI/Nursing mother/24 years/Oranyan)

The views of expressed by most people showed that the perceptions of women were considered more important in children's healthcare in line with the position of Asakitipi (2010) that there is a relationship between mothers' perceptions of diarrhoea and treatments sought.

\section{Perceptions of feeding patterns during and after Childhood Diarrhoea Episodes}

The study attempted to find out the feeding patterns adopted for children in most households. Findings showed that, generally there was neither special food nor feeding pattern adopted for children as a preventive measure against incidence of childhood diarrhoea. This was the submission of most participants at FGD session with illiterate mothers:

I give my child whatever we eat at home. This will also prevent the child from stealing or looking at other people's food. (FGD/Illiterate mothers/Aremo)

Once a child is of age it is better to introduce the child to the home meals for proper growth, and in line with the tradition of the people. We have our own foods which are meant for children like Ogi (pap) and Amala with ewedu or ila (Yam flour and okra soup). (FGD/Illiterate fathers/Aremo)

Participants were asked about their perceptions of food and feeding practices for children during and after childhood diarrhoea. Participants at separate FGDs for male and female, literate and illiterate, generally believed that certain foods should be avoided to prevent a child from having diarrhoea, while some 
other foods should be avoided during and after diarrhoea episodes. The view of a father, presented here, summed up this position:

There are certain foods like beans, Obe gbegiri (beans stew), Asaro (porridge), milk and vegetables that should not be given to a child with diarrhoea, especially in the night. They disturb the stomach which often needs to be rested in the night. (FGD/ Illiterate fathers/Orita-aperin)

This position suggested that consumption of certain foods could worsen the illness condition of children with diarrhoea. It was corroborated by a nursing mother that:

Ewa (beans), Akara (fried beans cake), Asaro (porridge), Moinmoin (beans cake), milk and vegetables are not too good as they worsen diarrhoea cases. (IDI/Nursing mother/30 years/Iworoad)

Most participants identified beans, vegetables and beans-related food items as capable of worsening diarrhoea episodes amongst children. However, the matron of one of the public hospitals interviewed disagreed with the people. She explained that:

Generally, most people believe that certain foods should be avoided when a child has diarrhoea but it is often discovered that protein foods are the most listed. This, to me, is because people find them not as sweet and cheap as carbohydrates. Our people love heavy meals. Again people know so little about nutritional values of their food, and we often educate them at various antenatal and post-natal meetings. (KII/Matron/Public hospital/Iworoad)

A community leader in Basorun reiterated the belief of the people that influenced their food culture and what was being done to change this perception:

The general belief about certain food taboos is common among our people. But recent health talks in hospitals are changing that perception. We pray our people will know that. Usually, the people believe in giving solid food like Gaari to the sick children, to help stop stooling. (KII/Community leader/Basorun)

On the need for breast feeding during childhood diarrhoea for children not yet weaned, some of the interviewees expressed divergent views on what could impede their adoption of exclusive breast feeding, the most prominent of which was the view expressed by a nursing mother:

The child's teeth will be hot and aching hence I cannot practise exclusive breast feeding until full recovery when the child will 
be comfortable to suck the milk. (IDI/Nursing mother/35 years/Aremo)

A community leader expatiated on why women would not want to breast feed a child during diarrhoea episode:

Women sometimes stop breast feeding a child, especially when the child has developed teeth. They sometimes fear that the child could even bite them, while attempting to scratch the teeth against their breasts. (KII/Community leader/Ojaagbo)

On number of times a child infected with diarrhoea was fed, the response below during an IDI with a mother of an infected child rightly summarised the people's belief:

A kii da igbe ti omo ko ba ya idoti inu etan. O si ye ki a din iye igba ti o ba njeun ku ki o ma baa tun da kun igbe yiya naa.

Translated thus:

We don't stop a child from stooling until all the dirty things in the stomach have been removed al. And it is also necessary that we reduce the feeding times or rate so that it will not worsen the passage of stool. (IDI/Nursing mother/28 years/Ojaagbo)

The position of some community leaders about feeding patterns adopted for children in the community during and after diarrhoea episodes summarised the people's perceptions about feeding practices in home management of diarrhoea:

You don't give a child with diarrhoea infection certain foods that are capable of aggravating the situation such as milk, vegetables, beans and sugary foods. Then you should starve the stomach a little by either skipping meals or even fasting for part of the day in case of adults. (KII/Community leader/Basorun)

If the stomach is too full, this may result in frequent passage of watery stools hence you may need to starve the stomach or reduce the quantity of food being given to the infected child. (KII/Community leader/Ojaagbo)

On perceived type of food, a child should eat after experiencing diarrhoea episode, many of the participants were of the opinion that solid and carbohydrate foods should be given to help the child regain strength and block the alimentary system. This perception is summarised by the opinion of a woman during an IDI that: 
It is better one allows the child to pass out the wastes as stool. However, if one must stop it, it is better to give the child solid food like eba or gaari (local staple from cassava) which will help block the system. And also reduce watery substances in the intestine. (IDI/Nursing mother/32 years/Orita-aperin)

However, this position was contrasted by a matron (Nurse) at a public hospital who stated that:

There is no need to stop feeding on nutritional food items like vegetables, beans and plantain. These are food items needed for recovery by the child, except where the child shows signs of allergy to any of the items. Most of these taboos are due to beliefs and tradition. (KII/Matron/Public hospital/Aremo)

\section{Discussion of Findings}

Findings from this study showed that not all types of diarrhoea were recognised as illnesses, and this influenced decision making on treatment option. This is in line with the findings of Omotade, Adeyemo, Kayode and Oladepo (2000) in a similar research. The results showed that people's culture influenced their perceptions of causes of childhood diarrhoea. The concept of disease varies across cultures in line with the assertion of Asakitipi (2007) which recognises the position of culture as a determinant of treatment choice for childhood diarrhoea. Findings from this study correlates with previous studies which posited that food taboos are often culturally situated (Gittelsohn and Vastine, 2003).

Further on perceived causes of diarrhoea, findings from the study confirmed previous positions of Asakitipi (2004) and Jegede et al. (2002) about the belief among Yoruba women that advent of teeth often causes diarrhoea through the pain that is associated with their eruption. This condition, it is believed, usually causes a child's body temperature to rise, which upsets the stomach and subsequently causes diarrhoea. This perhaps influenced the mothers' position on the need not to stop stooling, considered natural cleansing process. Mother's education influenced perception and incidence of repeat diarrhoeal episodes in line with findings of Yilgwan and Okolo (2012) that showed the important relationship between diarrhoeal morbidity and low maternal education, nonexclusively breastfed infant, and previous diarrheal episode in a sibling. This has far reaching consequences on the survival of under-fives.

The perception about the causes of diarrhoea is seen as the reason for neglect of the sick child and thus explains the high mortality rate amongst the under-fives. Some of the mothers interviewed did not believe in the fatality of diarrhoea in line with similar findings by Asakitipi (2010). The finding is in line with the supposition that least modernized groups are more likely to indulge in socio-cultural practices detrimental to children survival (Gyimah, 2002). 
Findings from the study were consistent with Weberian social action theory in which actors have choices or alternatives which are often subjective thus expressing individualism rather than collectivism orientation. Mothers' perception of diarrhoea as illness is socially constructed in the process of interactions with others in the society. Also choice of feeding patterns during and after diarrhoea episodes were found to be influenced by mothers' social status, the significant others (other family members and neighbours) and prevailing culture of the people. The perceptions of educated women were different from those of the illiterates as presented during FGD sessions.

Findings revealed mothers' low knowledge of feeding patterns required for children during and after diarrhoeal episodes. This agreed with the position of Adhikari (2010). The health seeking behaviour of women with diarrhoea infected children were found be influenced by culture and beliefs of the society. The poor feeding patterns adopted by mothers, according to Sharafkhani et al. (2011) can result into poor nutrition which can lead to reduced immunity, impaired physical and mental development and reduced productivity.

Findings from the study further revealed that there were food proscriptions which have cultural origins. There were food restrictions as some food items were considered harmful to a child with diarrhoea. Most of the foods listed were those needed for recovery from the illness and enhance healthy growth of the child. These include protein food, sugary food, oily food and leafy vegetables, in support of similar findings by Khalil, Mirsham, Zarghami, Rjabia and Farahmand (2013). The number of feeding times of children with diarrhoea was found to have been reduced with most parents adopting skipping of meals which could be attributed to influence of culture and tradition of the people. Findings showed that some of the household treatments patterns were largely influenced by the belief of the people, especially about food, and the environment in which the mothers live. This corroborated earlier findings by Asakitipi, (2010) that mother's perception of diarrhoea has a significant relationship with choice of treatments for childhood diarrhoea.

The results obtained from the field further confirmed that mothers' sociodemography is a major determinant of the feeding patterns adopted for children during diarrhoea episodes. Women are therefore important actors, as mothers, in determining the health status of children, and treatment choice in case of disease attack (Iruonagbe, 2010).

\section{Conclusion}

The study revealed the relationship between perceptions about diarrhoea and the people's culture in determining treatment choice. It showed that for effective treatment of diarrhoea to be achieved mothers' knowledge will have to be enhanced. The study therefore concluded that mothers' social status and perceptions, as influenced by significant others and the prevailing culture of 
the people, are strong determinants of the feeding patterns adopted for children with diarrhoea episodes.

The study concluded that mothers still have misconceptions about feeding practices to be adopted for children with diarrhoea episodes. This poor knowledge of feeding practices in home management of diarrhoea revealed the low knowledge in nutritional education amongst mothers. Mothers as caregivers are major determinants of type of food to be given to children. Their perceptions of feeding practices to be adopted in home management of diarrhoea may have effect on the diseases burden of the nation and threaten realisation of the targets on maternal and child health in the Sustainable Development Goals (SDGs), and the goals of United Nations Global Strategy for Women's and Children's health, with the specific goals of reducing mortality from diarrhoea in children less than 5 years of age fewer than 1 per 1000 live births.

\section{Recommendations}

The study therefore concluded on the need for improved nutritional education and health talks on home management of diarrhoea, since the household remains the primary source of food and health care for children, and mothers are custodians of the food and health needs of children. This will help to correct the myth around the proscriptions of certain foods for children during and after diarrhoea episodes. There should be advocacy against injurious feeding practices, which are based on food beliefs and food culture, towards enhancing the success of primary health care programmes (PHC).

The current free-feeding (home feeding) programme in Nigerian primary schools should be extended to include provision of free health care to children. The need to be cautious in observing sanitation in food environment should be emphasised. There should be a more rigorous health campaigns in all communication media to sensitize mothers to the dangers in adopting poor feeding practices in home management of diarrhoea, to enhance full recovery of the sick children.

\section{References}

Adhikari, R.K. (2010) Food Utilization Practices, beliefs and taboos in Nepal; An overview. Published by United States Agency for International Development, USAID (2010). Accessed on www.dec.org.

Ansari, M., Ibrahim, M.I.M., Hassali, M.A., Shankar P.R., Koirala, A., Thapa, N.J. (2012) Mother's beliefs and barriers about childhood diarrhoea and its management in Morangi district, Nepal. https://bmcresnotes.biomecentral.com/articles/accessed on may, 2017.

Aremu, O., Lawoko, S., Moradi, T., Dalal, K. (2011) Socio-economic determinants in selecting childhood diarrhoea treatment options in SubSaharan Africa: A multilevel model. 
Asakitikpi, A.E. (2004) Risk Factors Influencing the Incidence of Childhood Diarrhoea in Ibadan Metropolis, Nigeria. A doctoral dissertation in the Department of Sociology, University of Ibadan.

Asakitipi, A.E. (2007) An Interrogation of Diarrhoea Concept among Yoruba Women in Ibadan Metropolis. Nigeria Nordic Journal of African Studies, 16(1): 64-74.

Asakitipi, A.E. (2010) Acute Diarrhoea: Mother's knowledge of ORT and it's usage in Ibadan Metropolis Nigeria. EtnoMed, 4(2).

Children's Health Watch (2012) Food insecurity threatens children's health and wellbeing, SNAP Vaccine. www.childrenshealthwatch.org/.../ accessed in May, 2016.

Collise, N. and Goduka, N.I. (2012) Treatment of Diarrhoea in Rural African Communities: An Overview of Measures to Maximise the Medicinal Potentials of Indigenous Plants: Int $J$ Environ Res Public Health. November. 9(11): 3911-3933. Published online on http://www.ncbi.nlm.nih.gov/pmc/articles/PMC3524604/ accessed on August, 2016.

Food and Agriculture Organisation (FAO) (2011) Food safety for whom? Corporate wealth versus people's health. www.grain.org accessed on 22/ $17 / 2012$.

Fayehun, O. and Omololu, O. (2008) Prevalence and Treatment of Childhood Diarrhea among Nigerian Ethnic Groups. The Nigerian Journal of Sociology and Anthropology, Vol. 7.

Ghasemi, A.A., Talebian, A., Masaoud, A.N. and Mousari, G.A. (2013) Knowledge of mothers in management of diarrhoea in under five children in Kasham, Iran. Nurse Midwivery Study. 1: 158-162.

Gittelsohn, J. and Vastine, A.E. (2003) Sociocultural and Household Factors Impacting on the Selection, Allocation and Consumption of Animal Source Foods: Current Knowledge and Application. Downloaded from jn.nutrition.org at Nigeria: ASNA.

Gyimah, S.O. (2002) Ethnicity and infant mortality in sub-Saharan Africa: The Case of Ghana. Population Studies Centre Discussion Paper 02-10. The University of Western Ontario, and London, Ontarioin.

Iruonagbe, T.C. (2010) Gender Equity and Food Security: Lessons from Ozalla Community, Edo State, Nigeria. African Renaissance, 7(3-4): 89-.

Jegede, A.S., Oke, E.A., Oluwatosin, B., Ajala, A.S. and Adejumo, P.O.M. (2002) Food taboos and Management of Diarhoea Diseases among children in Ibadan, Nigeria. Unilag Sociological Review (USR), 3: 47-63.

Khalil, M., Mirsham, M., Zarghami, A., Rjabia, M. and Farahmand, M. (2013) Maternal knowledge and Practice Regarding Childhood Diarrhoea and Diet in Zahedan, Iran. JhHealthscope, 2(1): 19-24.

Merga, N. and Alemayehu, T. (2015) Knowledge, Perception and Management Skills of mothers with under five children about diarrhoeal disease in 
indigenous and resettlement communities in Assova District, Western Ethiopia. Journal of Population and Nutrition, 33(1): 20-30.

Mwambete, K.D. (2010) Joseph R. Knowledge and perception of mothers and caregivers on childhood diarrhoea and its management in Temeke municipality, Tanzania. Tanz. J. HealthRes, 12: 1-9. Italian J. Paed. 37:18.

Ogunbiyi and Akinyele (2010) Knowledge and belief of Nursing Mothers on Nutritional Management of acute diarrhoea in infants, Ibadan, Nigeria. African Journal of clinical and Experimental Microbiology, 5(1).

Okoronkwo, I., Onyia-Pat, J., Okpala, P., Agbo, M. and Ndu, A. (2014) Patterns of complementary and Alternative Medicines Use, Perceived Benefits and Adverse effects among Adults Users in Enugu Urban, Southeast, Nigeria. Evidence-Based Complementary and Alternative Medicine, Vol. 2014, Article ID 239372.

Omotade, O.O., Adeyemo, A.A., Kayode, C.M. and Oladepo, O. (2000) Treatment of childhood Diarrhoea in Nigeria: Need for Adaptation of Health Policy and programmes to cultural norms. Journal of Health and Population Nutrition, 18(3): 139-144.

Pantenburg, B., Ochoa, T.J., Ecker, L. and Ruiz, J. (2014) Feeding of young children during diarrhoea; Caregivers' intended practices and perceptions. Am. J. Trop Med. Hyg.

Sharafkhani, R., Dastgiri, S., Gharaaghaji, A.R. and Ghavamzadeh, S. (2011) Factors influencing household food insecurity status. Food and Nutrition Sciences, 2: 31-34.

Shuaib, K., Nigel, R. and Jan Van den B. (2004) Local Beliefs about Childhood Diarrhoea: Importance for Healthcare Research. Journal of Tropical Paediatrics.

United Nations Children's Fund, Unicef/WHO Final report (2009) Diarrhoea: Why children are still dying and what can be done. Geneva: United Nations Children's Fund/World Health Organization. www.who.int/maternal......

United Nations Children's Fund, Unicef/WHO/World Bank/United Nations Population Division (2015).

United Nations Development Programme (UNDP) (2010) What Will It Take to Achieve the Millennium Development Goals? An International Assessment 2010, UNDP; UN Children's Fund (UNICEF). www.unicef. org.

World Health Organisation (WHO)/UNICEF (2003) Joint statement: Clinical Management of acute diarrhoea. Reprinted August, 2004.ENAcute_Diarrhoea_reprint.pdf accessed on www.unicef.org.publica -tions.filesE...

World Health Organisation (WHO) (2007) Diarrhoea case management in low and middle income countries- an unfinished agenda. Bulletin of the World Health Organisation. 
World Health Organisation (WHO) (2013) Priority Medicines for Mothers and Children 2011, 3rd edition. http://www.who.int/medicines/publications/A4 prioritymedicines.pdf (Accessed in March 2013).

Yilgwan, C.S. and Okolo, S.N. (2012) Prevalence of diarrhoea disease and risk factors in Jos University Teaching Hospital, Nigeria: Ann Afr Med. OctDec, 11(4): 217-21. doi: 10.4103/1596-3519.102852. 